\title{
Carcinoid tumour of the cervix
}

\author{
E. DAw \\ M.B., Ch.B., M.R.C.O.G.
}

North Manchester General Hospital

\begin{abstract}
Summary
A case of non-metastatic carcinoid tumour of the cervix is described from a patient without the carcinoid syndrome. The rarity of carcinoid tumour involving gynaecological structures other than the ovaries is discussed and a brief review of carcinoid disease presented.
\end{abstract}

\section{Introduction}

The infrequent occurrence of carcinoid tumour of the cervix merits reporting the present case. Carcinoid disease is found in $0 \cdot 14-0.34 \%$ of all post-mortem examinations (Ritchie and Stafford, 1944). In most cases, the tumours arise from the gastro-intestinal tract, although a significant number have been reported as arising in the ovary, especially within dermoid cysts (Haines, 1971).

\section{Case history}

A pale 50-year-old woman was admitted as an emergency because of profuse vaginal bleeding following 5 months' amenorrhoea. There was no significant past gynaecological history. The patient had had one uncomplicated pregnancy 30 years before.

The cervix was stony-hard with a polyp, which was friable and bled on slight contact on the anterior lip. The polyp measured $6 \times 4 \times 3 \mathrm{~cm}$.

Laboratory examination confirmed the anaemia. The haemoglobin was $7.4 \mathrm{~g} / \mathrm{dl}$ and the patient was transfused with $1000 \mathrm{ml}$ of whole blood.

Examination under anaesthesia was performed 3 days after admission, when the friable polyp was removed. Dilatation of the cervix and curettage of the uterine cavity were also performed and a few scraps of endometrium were obtained.

Histologically the endometrium was in the proliferative phase and the flesh-coloured polyp was 'an unusual small-cell malignant tumour resembling an undifferentiated squamous cell carcinoma with a distinct pattern of a carcinoid tumour'. The cells were intensely eosinophilic (Fig. 1). Excision did not appear to te complete. Silver stains failed to demonstrate 5-hydroxytryptamine.

Correspondence: Dr E. Daw, The North Manchester General Hospital, Crumpsall, Manchester M8 6RB.

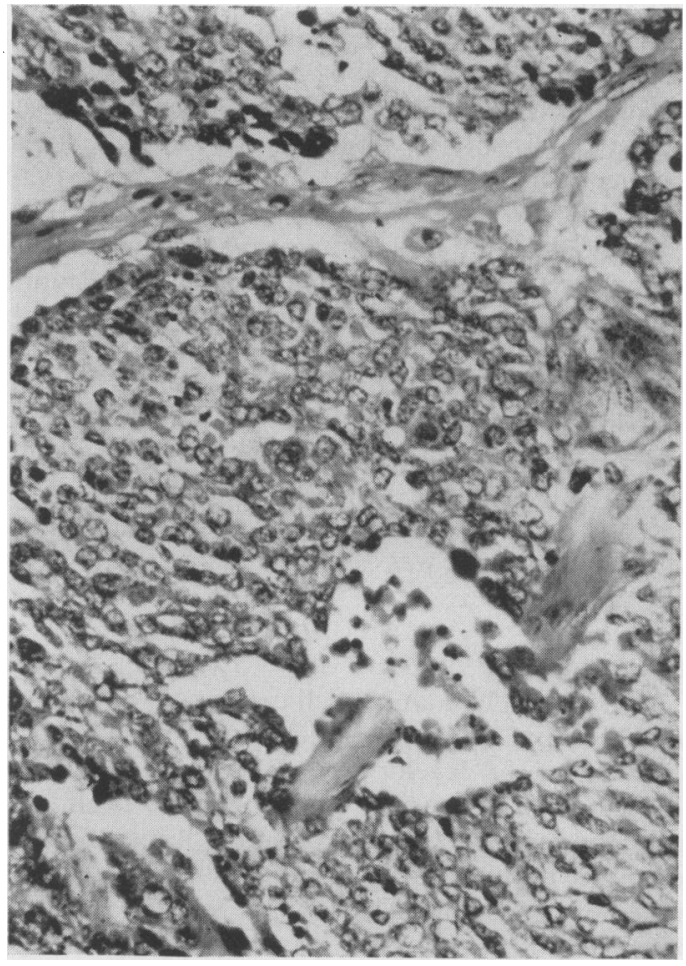

Fig. 1. Cervical polyp with distinct patterns of carcinoid tumour $(\mathrm{HE}, \times 24)$.

Radiographic studies, including an upper gastrointestinal series, intravenous pyelogram and chest $\mathrm{X}$-ray, were within normal limits. 5-Hydroxyindol acetic acid determinations on 24-hr urine specimens were 45 and $40 \mu \mathrm{mol} / 24 \mathrm{hr}$, within normal limits.

The patient was re-admitted to hospital approximately 1 month after the first operation when, in view of her age and as the lesion was thought not to be completely excised, total hysterectomy was performed. In view of the possibility of the lesion being secondary, bilateral salpingo-oophorectomy and appendicectomy were also performed, although at operation no tumour was palpated in these structures nor in the large or small intestine, regional lymph nodes or liver. Her postoperative recovery was unremarkable. The patient has been seen for follow-up 
6 weeks and 3 months postoperatively. She remains well (1 year later) with no sign of recurrence or secondary.

There was no histological evidence of residual tumour in the cervix or gynaecological tumour in either of the ovaries. There was no evidence of carcinoid tumour in the appendix.

\section{Discussion}

Carcinoid tumours with metastasis to the cervix and corpus uterus have been reported (Post et al., 1966). They most commonly originate in the argentaffin or Kultschitzky cells of the appendix and ileum, but may also arise elsewhere in the gastrointestinal tract and in the tracheobronchial tree and genital tract, where the epithelium has a biological and histopathogenetic similarity (Mikhailov, Terekhova and Gevorkyan, 1963). They are regarded as low-grade, slow growing malignancies that may metastasise locally to regional nodes, the liver or more distant organs. Over $90 \%$ occur in the appendix but metastases are uncommon as the patient usually has symptoms of acute appendicitis and the tumour is removed before spread has occurred.

Some carcinoid tumours present as the carcinoid syndrome, usually when an intestinal carcinoid tumour has metastasised to the liver, but also rarely with other sites such as in the bronchus. As the metastatic tissue in the liver secretes directly into the hepatic veins, considerable amounts of 5-hydroxytryptamine (5-HT) escape destruction and account for the clinical symptoms of 'carcinoid syndrome' (facial flushing, abdominal pain, intestinal hypermotility and diarrhoea, cardiac valvular lesions, bronchoconstriction and peripheral oedema). The argentaffin cells appear to be the store of the 5-HT; the Kultschitzky cells may be the store of the various kinins associated with the syndrome. More recently prostaglandins have been isolated from carcinoid tissue but their role in the syndrome remains to be evaluated (Delmont and Rampal, 1975).

The finding of excessive 5-hydroxyindolacetic acid (5-HIAA), a metabolite of 5-HT, in the urine is diagnostic of carcinoid syndrome if other causes are excluded (Page et al., 1958). However, as in the present case, a normal excretion level of 5-HIAA may be found (see also Davis and Rosenberg, 1959). It may be postulated that in these cases the carcinoid tumour has fewer argentaffin cells.

\section{Acknowledgment}

I thank Dr T. de Kretzer for the pathological reports, the Librarian at the Royal College of Obstetricians and Gynaecologists for her help and Mrs M. Tudor for typing the manuscript.

\section{References}

Davis, R.B. \& Rosenberg, J.C. (1959) Carcinoid syndrome with normal 5-HIAA excretion. Journal of Laboratory and Clinical Medicine, 54, 804.

Delmont, J. \& Rampal, P. (1975) Prostaglandins and carcinoid tumours. British Medical Journal, 4, 165.

HAINES, M. (1971) Carcinoid tumours of the ovary. Journal of Obstetrics and Gynaecology of the British Commonwealth, 78, 1123.

Mikhailov, V.P., Terekhova, A.A. \& Gevorkyan, G.C. (1963) Intra-epithelial cancer of cervix uteri (morphological, clinical picture, treatment). Acta Unionis internationalis contra cancrum, 19, 1415.

Page, I., Corcoran, A.C., Udenfriend, S., Szoedsma, A. \& Weissbach, H. (1958) Argentaffinoma as an endocrine tumour. Lancet, i, 198.

Post, R.C., Cohen, T., Blaustein, A.U. \& Shenker, L. (1966) Carcinoid tumor metastatic to the cervix and corpus uteri. Obstetrics and Gynecology. New York, 27, 171.

Ritchie, G. \& Stafford, W.T. (1944) Argentaffin tumors of the gastrointestinal tract. Archives of Pathology, 38, 123. 